Availability of new antiepileptic drugs. Cost-effectiveness as a reason for availability of new antiepileptic drugs is debated in Controversies in Neurology (Arch Neurol Feb 2000;57:272-275). Pierre-Louis SJC argues in favor of all new drugs being included in the Formulary, if only for use by specialists initially, provided cost-effectiveness has been addressed in trials. Bergen DC would restrict their availability according to cost-effectiveness based on meaningful studies. Hachinski $\mathrm{V}$ points out that in principle all AEDs should be available to those who can afford them, but in practice cost-effectiveness will be the determining argument for most.

As a group, new AEDs are more costly, they tend to have more specific sites of action and fewer adverse reactions, but their greater effectiveness is often uncertain. However, despite their immediate cost, the saving of expense in avoidance of hospitalization for side reactions of conventional drugs may prove cost-effective. For example, fosphenytoin is more expensive than generic phenytoin, but its recent introduction in ER treatment of seizures has appreciably lowered the frequency of complications. Similarly, the prompt administration of rectal diazepam has avoided prolongation of febrile and other acute seizures that may otherwise require hospitalization for status epilepticus.

\title{
RISKS OF AED WITHDRAWAL AFTER EPILEPSY SURGERY
}

The frequency of seizure recurrence and outcome factors associated with antiepileptic drug (AED) withdrawal following successful epilepsy surgery were evaluated retrospectively in 210 patients treated between 1989 and 1993 at the Mayo Clinic, Rochester, MN. The mean age was 32 years; 6 were children younger than 12 years, and 19 were adolescents (12-18 years). Ninety percent had a temporal lobe resection and in $10 \%$ resection was extratemporal. Medical therapy was reduced in 96 and discontinued in 84 patients. At 2 and 5 year follow up, seizure recurrence rate after complete AED withdrawal was $14 \%$ and $36 \%$, in contrast to only $3 \%$ and $7 \%$ of 30 patients who continued AEDs unchanged. Seizure-free patients who partially reduced AEDs had an intermediate rate of recurrence between that of controls and those with complete AED withdrawal. After AED withdrawal, prevalence of seizure recurrence was higher in patients with normal preoperative MRI studies (40\%) compared to those with focal pathology (20\%), including hippocampal atrophy (NS). Factors not predictive of seizure recurrence after AED withdrawal included electrocorticography at operation, extent of resection, EEG postoperatively, and seizure-free duration after surgery. (Schiller Y, Cascino GD, So EL, Marsh WR. Discontinuation of antiepileptic drugs after successful epilepsy surgery. Neurology January (2 of 2) 2000;54:346349). (Reprints: Dr Yitzhak Schiller, Department of Neurology, Rambam Medical Center, PO Box 9602, Bat-Galim Haifa 31096 Israel).

COMMENT. Long-term seizure recurrence rate following AED withdrawal in patients, principally adults, successfully treated with surgery may be as high as $36 \%$ with complete AED withdrawal, compared to $14 \%$ following partial AED withdrawal, and $7 \%$ when AED therapy is unchanged. A normal preoperative MRI is a risk factor for higher seizure recurrence than in patients with well defined pathology. When seizures recur after AED withdrawal, renewal of therapy is usually successful, irrespective of the seizure-free duration after surgery. The results of this study are inconclusive regarding a general policy for AED withdrawal after successful epilepsy surgery, and should not be applied to children who formed only $12 \%$ of the study group. Each patient must be considered as an individual when evaluating the pros and cons of AED therapy. 